\title{
rAAV-compatible MiniPromoters for restricted expression in the brain and eye
}

\author{
Charles N. de Leeuw ${ }^{1,2}$, Andrea J. Korecki ${ }^{1}$, Garrett E. Berry ${ }^{3}$, Jack W. Hickmott ${ }^{1}$, Siu Ling Lam ${ }^{1}$, Tess C. Lengyell ${ }^{1}$, \\ Russell J. Bonaguro', Lisa J. Borretta', Vikramjit Chopra ${ }^{4}$, Alice Y. Chou', Cletus A. D'Souza ${ }^{4}$, Olga Kaspieva', \\ Stéphanie Laprise ${ }^{1}$, Simone C. McInerny ${ }^{1}$, Elodie Portales-Casamar ${ }^{1}$, Magdalena I. Swanson-Newman', \\ Kaelan Wong ${ }^{1}$, George S. Yang ${ }^{4}$, Michelle Zhou', Steven J. M. Jones ${ }^{2,4,5}$, Robert A. Holt 2,4,5, ${ }^{1}$ Aravind Asokan ${ }^{3}$, \\ Daniel Goldowitz ${ }^{1,2}$, Wyeth W. Wasserman ${ }^{1,2}$ and Elizabeth M. Simpson ${ }^{1,2,6^{*}}$
}

\begin{abstract}
Background: Small promoters that recapitulate endogenous gene expression patterns are important for basic, preclinical, and now clinical research. Recently, there has been a promising revival of gene therapy for diseases with unmet therapeutic needs. To date, most gene therapies have used viral-based ubiquitous promoters-however, promoters that restrict expression to target cells will minimize off-target side effects, broaden the palette of deliverable therapeutics, and thereby improve safety and efficacy. Here, we take steps towards filling the need for such promoters by developing a high-throughput pipeline that goes from genome-based bioinformatic design to rapid testing in vivo.

Methods: For much of this work, therapeutically interesting Pleiades MiniPromoters (MiniPs; 4 kb human DNA regulatory elements), previously tested in knock-in mice, were "cut down" to $\sim 2.5 \mathrm{~kb}$ and tested in recombinant adeno-associated virus (rAAV), the virus of choice for gene therapy of the central nervous system. To evaluate our methods, we generated 29 experimental rAAV2/9 viruses carrying 19 different MiniPs, which were injected intravenously into neonatal mice to allow broad unbiased distribution, and characterized in neural tissues by X-gal immunohistochemistry for icre, or immunofluorescent detection of GFP.

Results: The data showed that 16 of the 19 (84\%) MiniPs recapitulated the expression pattern of their design source. This included expression of: Ple67 in brain raphe nuclei; Ple155 in Purkinje cells of the cerebellum, and retinal bipolar ON cells; Ple261 in endothelial cells of brain blood vessels; and Ple264 in retinal Müller glia.
\end{abstract}

Conclusions: Overall, the methodology and MiniPs presented here represent important advances for basic and preclinical research, and may enable a paradigm shift in gene therapy.

Keywords: rAAV Gene therapy, Raphe nuclei, Purkinje cells, Retina, Cornea

\section{Background}

Interest in the identification of small promoters for recapitulating unique gene expression patterns is longstanding, as such promoters are widely used in basic and preclinical research [1-6]. Recently, there has been a promising revival of gene therapy for diseases with unmet treatment needs [7].

\footnotetext{
* Correspondence: simpson@cmmt.ubc.ca

${ }^{1}$ Centre for Molecular Medicine and Therapeutics at the Child \& Family Research Institute, University of British Columbia, 950 W 28 Ave, Vancouver, BC V5Z 4H4, Canada

${ }^{2}$ Department of Medical Genetics, University of British Columbia, Vancouver, BC V6H 3N1, Canada

Full list of author information is available at the end of the article
}

Most avant garde of these are Imlygic ${ }^{\circ}$ and Glybera ${ }^{\oplus}$, virusbased gene therapies approved for marketing by the U.S. Food and Drug Administration in 2015, and the European Medicines Agency in 2013, respectively. To date, these and most gene therapies have used viral-based ubiquitous promoters. However, it has been recognized by leaders in the field that "a more deliberate process of promoter selection may be beneficial in the long run by determining the minimum promoter activity that is necessary to tailor AAVmediated gene therapy to a particular neurological disease." [8]. It is anticipated that promoters that restrict expression to target cells will minimize off-target side effects, 
broadening the palette of deliverable therapeutics, and thereby improving safety and efficacy [8-10]. Recombinant adeno-associated virus (rAAV) is the gene-therapy vector of choice for many clinical applications, especially in non- or slowly-dividing cells, which are typical of the CNS [11-17]. However, the limited AAV-packaging capacity of $\sim 4.9 \mathrm{~kb}$ [18] leaves very little space for a promoter. In contrast, mammalian promoters are generally large and complex, with cis-regulatory modules (CRMs) dispersed throughout the gene. Furthermore, identifying CRMs and predicting their function remains a major challenge $[2,19-21]$. In this work, we take steps towards filling the research and expanding clinical need for small promoters with restricted expression by developing them for use in rAAV for the brain and eye.

We accomplished this goal by building upon our previously developed "MiniPromoters" (MiniPs), human DNA elements designed to drive expression in restricted cell types [22-25]. Previously, we bioinformatically parsed the entire human genome to identify genes suitable for MiniP design [24]. Further selection steps included genes with therapeutically interesting conserved expression patterns in human and mouse adult brain [26], and conserved computationallypredicted candidate regulatory regions (RRs). In general, MiniP designs were $\sim 4 \mathrm{~kb}$, consisting of a PROM $(\sim 1.1 \mathrm{~kb}$ at the transcription start site; range 254-3,536 bp) and multiple CRMs ( 900 bp each; range 28-2,951 bp), which were usually non-contiguous and typically repositioned 5 ' of the PROM. We named our MiniPs sequential by "Ple" number (for Pleiades Promoter Project). Initially, MiniPs were tested driving a reporter after being knocked-in, single-copy, sitespecific $5^{\prime}$ of the mouse Hprt gene. Key features of this strategy included: 1) a large-scale pipeline, to reduce the impact of the expected high failure rate in promoter design; 2) physiologically relevant expression, potentially more suitable for therapeutic delivery; 3) human DNA, presumably decreasing our success when initially tested in mice, but favoring translation to humans if positive; and 4) the expectation that endogenous-like expression in mice best predicts a similar expression will occur in humans. In total, 45 such positive MiniPs have been developed [22, 24] and made available to the research community (www.addgene.org and www.jax.org).

However, we anticipate that not all MiniPs, which functioned as single-copy site-specific knock-ins (KIs) in the mouse genome, will also function specifically in rAAV. For example: 1) multiple virus copies may increase and thereby broaden expression; 2) developmentally-established epigenetic marks may not be acquired by a promoter introduced after birth; and 3) most of the original Pleiades promoters will need to be "cut down" to be useful in rAAV, which may inadvertently remove essential elements. However, we have previously tested three MiniPs developed in mice (one unaltered and two cut downs) in rAAV2 and were encouraged by their success [22]. On the other hand, this data set was small; thus, to draw a stronger conclusion and to provide more resources for the field, in this study we have evaluated a much larger set of 19 MiniPs. For this evaluation, we established a two-step, relatively large-scale high-throughput screening system for human MiniPs packaged in rAAV and tested in mouse. After promoter design, DNA was synthesized, cloned into our "plug and play" rAAV2 genome plasmid, packaged as rAAV2/9 for broad tropism, and injected intravenously into neonatal mice, with histological analysis of expression in adult brain and eye [27]. The first step of the screening uses a highly-sensitive historical-indirect reporter system, in which the MiniP drives icre (improved cre recombinase [28]) in a cre-reporter mouse. In the second screening step, representative successful promoters were recloned driving the direct reporter emerald green fluorescent protein (EmGFP) [29] and the assay performed again. By undertaking these studies with the smallest and thus most challenging virus, rAAV, we anticipate that successful promoters will have a wide use in other gene therapy modalities such as lentivirus, adenovirus, retrovirus, herpes simplex virus, plasmids, and nanoparticles.

\section{Results and Discussion \\ Updated MiniPromoter virus pipeline allows for rapid construct screening}

We had previously scored every gene in the human genome for suitability for MiniP design by assessing regulatory resolution, a metric that favors small genes with clearly defined conserved regions [24]. MiniPs were initially designed based on information in primary literature, transcription start sites, sequence boundaries, phylogenetic footprinting, and transcription factor binding site predictions [24]. Now, as additional genome-wide datasets such as RNAseq, and new data and tools within projects such as FANTOM [30] and JASPAR [31] become publically available, they were exploited for bioinformatically-driven MiniP design and redesign. In addition, compared to our previous work, the current pipeline was intended to move directly from MiniP design to a virus-based assay, decreasing the costs associated with KI mouse engineering, greatly reducing turn-around time, and making sequential redesigns feasible in the future.

In establishing this pipeline, we wanted a system that transduced as many cells as possible and showed expression with a ubiquitous promoter in all relevant cell types, such that any observed restriction would be attributable to the promoter. Thus, we tested 15 control rAAVs including: self-complementary and single-stranded genomes; AAV9 and AAVrh.10 serotypes; CMV, smCBA, CBA, CAGGS, and promoterless constructs; hGFP, GFP, cre, icre, and EmGFP reporters; and the 3'-UTR woodchuck hepatitis virus post-transcriptional regulatory element (WPRE). We used WPRE mut6, a DNA element known to substantially increase expression levels but 

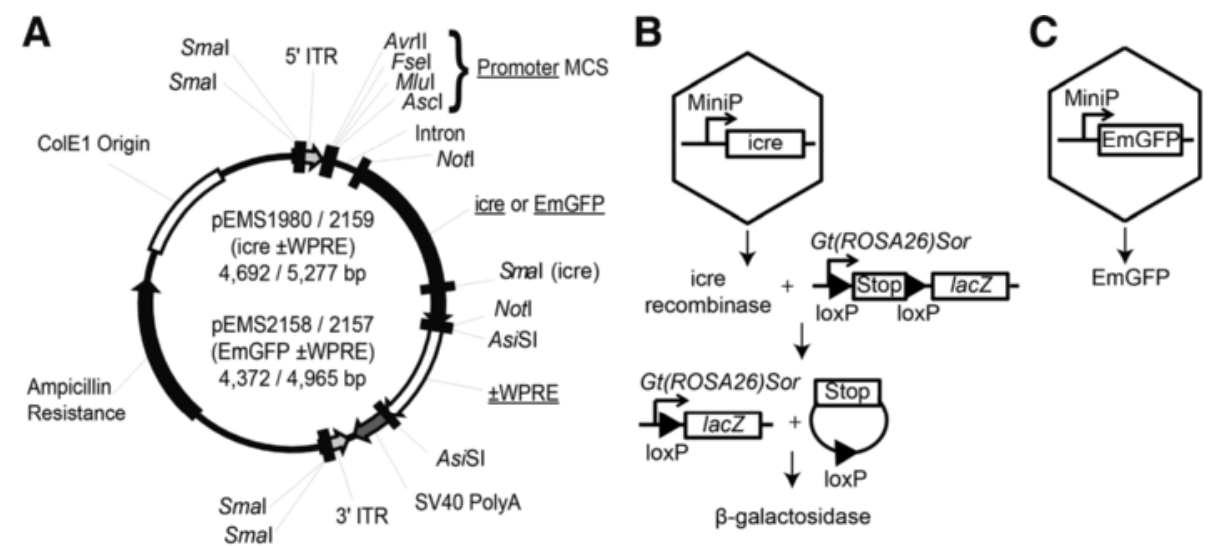

Fig. 1 "Plug and Play" rAAV2 genome plasmid used to clone MiniPromoters (MiniPs) upstream of icre or EmGFP enables high throughput pipeline testing. a Plasmids were generated containing either the icre or the EmGFP reporter. An AsiSl site flanks a removable WPRE. MiniPs were cloned at the MCS using a combination of the four available cut sites. The plasmids are subsequently used to generate single stranded rAAV. b Screening step one, an historical-indirect reporter system. MiniPs drive expression of icre, which in turn recombines the endogenous loxP sites and removes the stop sequence $5^{\prime}$ of the lac $Z$ gene, thus driving expression of $\beta$-galactosidase from the strong ubiquitous ROSA26 promoter. c Screening step two, a direct reporter system. MiniPs drive direct expression of EmGFP, which can be imaged by epifluorescence or signal amplified using antibodies. bp, base pairs; ITR, inverted terminal repeat; MCS, multiple cloning site; WPRE, woodchuck hepatitis virus posttranscriptional regulatory element

without promoter activity [32, 33]. Figure 1a depicts the "plug and play" rAAV2 genome plasmid we developed, with AAV2-based inverted terminal repeats (ITRs), an intron, either icre or EmGFP reporters, with or without WPRE. Restriction enzyme sites allowed for the rapid exchange of promoters, reporters, or removal of the intron and/or WPRE. Figure 1b depicts the first evaluation step of the pipeline, in which a MiniP drove icre, which permanently removed the "stop" from a mouse genomic locus where the ubiquitous ROSA26 promoter drove a "lox-stoplox-lacZ" allele, resulting in high-level expression of lacZ wherever and whenever icre was expressed. Thus, this step used an historical-indirect reporter of cre activity. Figure 1c depicts the second evaluation step of the pipeline, in which a positive MiniP was retested driving EmGFP, a direct reporter of MiniP expression, detected via epifluorescence or immunofluorescence staining.

\section{MiniPromoter selection surveyed a variety of design types}

Figure 2 lists the 29 experimental viruses generated for this project. The 19 MiniPs carried by these viruses were all chosen for potential therapeutic utility. The majority of our work consisted of $\mathrm{P} 0$ virus injections for both icre and EmGFP viruses. A subset of EmGFP constructs were also injected at P4, since it has been shown that the developmental age at which virus is delivered to the mouse eye determines the cell types most efficiently transduced [34].

Five previously designed MiniPs were tested "unaltered" in rAAV2/9: Ple34 (CLDN5 RRs), Ple67 (FEV RRs), Ple94 (GPR88 RRs), Ple155 (PCP2 RRs), and Ple198 (SLC6A4 RRs) [22, 24]. KI mouse data for three of these MiniPs (Ple34, Ple67, and Ple155) had been previously published
[22, 24], of which Ple67 was also known to drive expression in the eye following intravitreal rAAV delivery [22]. The remaining two MiniPs in this group (Ple94 and Ple198), were chosen despite negative KI mouse data due to their potential clinical impact, as we suspected detection was a limitation with a subset of our previously negative single-copy EGFP reporter KI mice [24]. Our hypothesis was that the multi-copy rAAV system would enhance low-level expression, allowing us to recapture previously "negative" MiniPs.

A further eight previously designed MiniPs, which were positive in KI mice, were chosen for "cut down" to $\sim 2.5 \mathrm{~kb}$ or smaller, after bioinformatics reanalysis identified regions that were less likely to contribute to regulatory function (Ple251 (C8ORF46 RRs), Ple253 (PITX3 RRs), Ple261 (CLDN5 RRs), Ple264 (NR2E1 RRs), Ple266 (S100B RRs), Ple267 (UGT8 RRs), Ple302 (DCX RRs), and Ple304 (OLIG1 RRs)). The majority of the original Pleiades MiniPs require such modification in order to be useful in the space-limited rAAV as they were initially $\sim 4 \mathrm{~kb}$ for KI mouse studies.

For two MiniPs (Ple301 (TNNT1 RRs) and Ple305 (OLIG1 RRs)) we undertook bioinformatically-driven "base-pair modifications" that were divergent from the reference human sequence, in favor of a transcription factor consensus sequence designed to strengthen the previously successful promoter. Thus, we included in the study Ple301, which carried two base-pair modifications compared to our previous Ple232, which was positive in KI mice [22]. The base-pair modifications were introduced to improve the core promoter region containing downstream core elements (DCEs), which are recognized by TFIID [35]. Similarly, Ple305 was a three 


\begin{tabular}{|c|c|c|c|c|c|c|c|}
\hline MiniPromoter-0RF & MiniPromoter Gene & MiniPromoter Design Source (PMID) & $\begin{array}{l}\text { MiniPromoter } \\
\text { Size (bp) }\end{array}$ & $\begin{array}{l}\text { Plasmid } \\
\text { pEMS } \\
\text { Number }\end{array}$ & $\begin{array}{l}\text { MiniPromoter } \\
\text { Design Type }\end{array}$ & $\begin{array}{l}\text { Expression Related } \\
\text { to Gene / Source }\end{array}$ & $\begin{array}{l}\text { Figure } \\
\text { Showing } \\
\text { Expression }\end{array}$ \\
\hline Ple34-minisOG & CLDNS & KI mouse $(18971253,24761428)$ & 3,845 & Unassigned & Unaltered & Related & 5 \\
\hline Ple67-icre & FEV & KI mouse and rAAV $(18971253,24761428)$ & 2,202 & 1990 & \multirow{4}{*}{ Unaltered } & \multirow{4}{*}{ Related } & (see EmGFP) \\
\hline Ple67-icre-WPRE & FEV & KI mouse and rAAV $(18971253,24761428)$ & 2,202 & 1991 & & & (see emGFP) \\
\hline Ple67-EmGFP & FEV & KI mouse and rAAV $(18971253,24761428)$ & 2,202 & 2114 & & & $4,6,55,56$ \\
\hline Ple67-EmGFP-WPRE & FEV & KI mouse and rAAV $(18971253,24761428)$ & 2,202 & 2113 & & & $4,6,55,56$ \\
\hline Ple94-icre & GPR88 & KI mouse NEGATIVE & 3,049 & 1995 & Unaltered & Related / NA & 3 \\
\hline Ple155-icre & $P C P 2$ & $\mathrm{KI}$ mouse (24761428) & 1,652 & 1985 & \multirow{4}{*}{ Unaltered } & \multirow{4}{*}{ Related } & (see EmGFP) \\
\hline Ple155-icre-WPRE & $P C P 2$ & KI mouse (24761428) & 1,652 & 1986 & & & (see EmGFP) \\
\hline Ple155-EmGFP & $P C P 2$ & KI mouse (24761428) & 1,652 & 2116 & & & $4,6,55$ \\
\hline Ple155-EmGFP-WPRE & $P C P 2$ & KI mouse (24761428) & 1,652 & 2115 & & & $4,6,55$ \\
\hline Ple198-icre & SLC6A4 & KI mouse NEGATIVE & 2,826 & 1997 & Unaltered & Related / NA & 3 \\
\hline Ple251-icre & C8ORF 46 & Ple17 KI mouse $(18971253,24761428)$ & 2,453 & 1977 & \multirow{2}{*}{ Cut down } & \multirow{2}{*}{ Related } & 3,56 \\
\hline Ple251-icre-WPRE & C8ORF 46 & Ple17 KI mouse (18971253, 24761428) & 2,453 & 1978 & & & 3,56 \\
\hline Ple253-icre & PITX3 & Ple160 KI mouse (18971253) & 2,484 & 1983 & \multirow{2}{*}{ Cut down } & \multirow{2}{*}{ Unrelated } & 56 \\
\hline Ple253-icre-WPRE & PITX3 & Ple160 KI mouse (18971253) & 2,484 & 1984 & & & S6 \\
\hline Ple261-icre & CLDN5 & Ple34 KI mouse $(18971253,24761428)$ & 2,963 & 1982 & \multirow{2}{*}{ Cut down } & \multirow{2}{*}{ Related } & (see EGFP) \\
\hline Ple261-EGFP & CLDNS & Ple34 KI mouse (18971253, 24761428) & 2,963 & Unassigned & & & 5 \\
\hline Ple264-icre & NR2EI & Ple140 KI mouse (18971253, 24761428) & 3,026 & 1981 & \multirow{2}{*}{ Cut down } & \multirow{2}{*}{ Related / Unrelated } & (see EmGFP) \\
\hline Ple264-EmGFP & NR2EI & Ple $140 \mathrm{KI}$ mouse $(18971253,24761428)$ & 3,026 & 2112 & & & 6,55 \\
\hline Ple266-icre & 51008 & Ple185 KI mouse (18971253) & 2,982 & 1994 & Cut down & Related & 3,6 \\
\hline Ple267-icre & UGT8 & Ple240 KI mouse (24761428) & 3,014 & 1996 & Cut down & Related & 3 \\
\hline Ple301-icre & TNNTI & Ple232 KI mouse (24761428) & 1,209 & 2026 & Base-pair modified & Related (muscle) & 53 \\
\hline Ple302-icre & $D C X$ & Ple53 KI mouse (24761428) & 2,359 & 2021 & Cut down & Unrelated / Related & 6,56 \\
\hline Ple303-icre & NOV & Ple134 a BAC KI mouse (24124870) & 3,087 & 2025 & New & Related & $3,6,56$ \\
\hline Ple304-icre & OLIGI & Ple151 KI mouse (18971253) & 2,596 & 1993 & Cut down & Related & 3 \\
\hline Ple305-icre & OLIGI & Ple304 rAAV, this study & 2,596 & 2031 & Base-pair modified & Related & 3 \\
\hline hs671-icre & $D P Y D-A S T$ (intragenic) & VISTA Ril mouse $(17130149,23253453)$ & 2,129 & 2024 & Literature & Unrelated & 54,56 \\
\hline hs1218-icre & $O T X 2-A S 1-E X O C 5$ (intergenic) & VISTA RI mouse (17130149) & 2,338 & 2023 & Literature & Unrelated & 54 \\
\hline PRS2/3:Con-icre & $D B H$ & Adenovirus ST injections in rat (15585414) & 987 & 2022 & Literature & Negative & None \\
\hline
\end{tabular}

Fig. 2 Twenty-nine rAAVs, carrying 19 MiniPromoters (MiniPs), from 17 gene/loci, resulted in 16 MiniPs with expression patterns related to the gene/source. MiniPs in black text drive icre; MiniPs in green text drive a green fluorescent protein; horizontal lines group MiniP constructs; note that the CLDN5-based MiniPs are separated in the table and thus highlighted in pink; BAC, bacterial artificial chromosome; bp, base pairs; EmGFP; emerald green fluorescent protein; icre, improved cre recombinase; Kl, knock-in; miniSOG, mini Singlet Oxygen Generator; NA, not applicable; ORF, open reading frame; PMID, PubMed ID number; rAAV; recombinant adeno associated virus; ST, stereotaxic; VISTA RI, random insertion from VISTA enhancer project; WPRE, woodchuck hepatitis virus post-transcriptional regulatory element

base-pair modification of Ple304 (a cut-down described above). The changes were designed to increase expression by strengthening the TATA-box and upstream B recognition elements (BREs) [36, 37].

Three promoters were also chosen from the "literature". Two were representative of the extensive community resource developed by the VISTA enhancer project, the main goal of which is to identify distant-acting noncoding regulatory elements that drive developmental gene expression in a region-specific manner [38]. VISTA enhancers are chosen using extreme evolutionary sequence conservation and/or ChIP-seq data, and then tested combined with a mouse minimal promoter (Hsp68) by random insertion in the mouse genome, with expression evaluated in E11.5 embryos. In our system, we tested two of their small human enhancers that had highly-specific brain expression: hs671 (forebrain; DPYD-AS1 intragenic genomic location), and hs1218 (midbrain; OTX2-AS1 EXOC5 intergenic genomic location). A third literature example was chosen due to its important therapeutic application in Parkinson disease. Although, in our previous work we were able to obtain regionalized expression with a $D B H$-based MiniP in KI mice, there was no clear overlap with tyrosine hydroxylase staining [24]. Thus, we choose to test PRS2/3:Con; a $D B H$-based promoter that had been successfully used in adenovirus in the rat adult brain [39].
Lastly, we computationally designed one completely new MiniP for direct testing in rAAV to assess our de novo design abilities. For this work, we chose NOV (nephroblastoma overexpressed gene). Previously, because $N O V$ had a poor RR score of 0.0439 primarily due to a large genomic region [24], despite clear TSS/promoter and RR elements, we had tested a retrofitted NOV BAC in a Hprt KI mouse with promising positive results [2]. Although a BAC construct may not delineate specific regulatory elements, it does provide genomic boundaries for the regulatory region. Thus, we designed a MiniP using elements within the NOV BAC, for testing in virus.

\section{Ubiquitous promoters demonstrate widespread transduction}

After an initial wide survey of methodologies, we focused on neo-natal intravenous delivery of singlestranded rAAV in AAV9, due to its excellent capability to transduce a variety of CNS cell types in our hands and as described in the literature [27, 40, 41]. To evaluate the reproducibility of these methods the expression of icre from the CAGGS and EmGFP from the CAGGS and smCBA ubiquitous promoters was analyzed. Consistent staining was seen, with positive staining in all brain regions, but reduced in areas such as the striatum, 
midbrain, ventral hindbrain, and Purkinje cells of the cerebellum (Additional file 1: Figure S1). With the direct EmGFP reporter, expression was markedly increased in intensity and in number of positive cells when combined with the WPRE.

These results are consistent with the literature on ubiquitous promoters, which indicates variation in their cell-type specific expression. The cell-type variation in ubiquitous promoter expression may result in inadequate reporting on the full spectrum of viral tropism for a particular AAV and delivery method [42-44]. For instance, the observation of poor expression in motor neurons with the CBA ubiquitous promoter, only to find strong expression when using a MeCP2-based promoter, which has neuronal expression in the CNS [43]. Thus, we propose that the true breadth of viral tropism in our system will be determined by the overlap of ubiquitous and specific promoters.

\section{6 novel MiniPromoters with consistent restricted expression}

In total, 19 Pleiades MiniPs were tested in rAAV2/9. Eighteen were tested driving icre in mice carrying a "lox-stop-lox-lacZ" allele, an historical-indirect reporter. Four of these were further tested driving either EGFP or EmGFP direct expression reporters. To avoid duplication, we only present the GFP data for these four. Finally, one Pleaides MiniP was tested driving miniSOG, a direct expression reporter. In all, we saw 16 MiniPs with restricted expression: 11 in the brain, 10 in the eye, and one in muscle.

The consistency of MiniPromoter constructs was assessed by analyzing expression patterns in multiple animals (Additional file 1: Figure S2). In the brain, all 19 MiniPromoter constructs showed $\geq 75 \%$ consistency with the figures presented ( $n \geq 4$ animals per construct). In the eye, 11/13 MiniPromoter constructs showed $\geq 70$ $\%$ consistency with the figures presented ( $n \geq 4$ animals per construct).

\section{MiniPromoters for the brain include restricted expression in Raphe nuclei and blood brain barrier}

Figure 3 presents the data for eight positive Pleiades MiniPs driving icre. We chose to first test MiniPs with the historical-indirect reporter system because of its high sensitivity, since any expression of icre, from the neonatal introduction of the virus to the adult harvest, would be captured by the strong genomic Gt(ROSA)26Sor promoter. Nevertheless, we were surprised by the generalized pattern of positive staining that was sparse, but widespread, and similar across all well-injected mice. This "background" may have been due to non-specific icre expression as a result of initial high viral copy number, the time required for appropriate epigenetic marks, and transcription factor binding. However, superimposed on this was the expected restricted expression for many of the MiniPs, suggesting this problem resolves. For example, two previously negative KI designs, Ple94 (GPR88 RRs) and Ple198 (SLC6A4 RRs), were positive and expressed in the striatum and thalamus respectively - as expected by the endogenous gene expression in development and/or the adult mouse [45-49]. We hypothesize that the KI mice may have expressed below detection levels either due to being single-copy, EGFP, or both. Notice also that the Ple94 MiniPromoter showed strong expression in the striatum, a region of weak expression with the ubiquitous promoter, thus expanding the characterization of the viral tropism in this screening system (Additional file 1: Figure S1). We also examined the effect of the WPRE on expression specificity, and noticed a very strong increase in the number of positive cells with the Ple251 (C8ORF46 RRs) MiniP. While Ple251-icre expressed in limited cortical layers, the hippocampus, and the zona incerta; the addition of the WPRE generalized expression to a near ubiquitous pattern. This result suggests that in this system, WPRE greatly enhances low-level and undetectable transcript levels. In addition, the current set includes four constructs that expressed in putative glial cells (Ple266 (S100B RRs), Ple267 (UGT8 RRs), Ple304 (OLIG1 RRs), and Ple305 (OLIG1 RRs)). Ple304 and Ple305 exhibited highly similar expression patterns, despite changes to the latter designed to strengthen expression. Finally, using our Ple303 MiniP, we demonstrate similar expression to the previous BAC construct [2], indicating success with our de novo design strategy.

Of course, negative, or partially negative results were also obtained. First, minor base-pair modifications were made to our previous Ple232 (TNNT1 RRs) MiniP, resulting in Ple301. In doing so, surprisingly, we lost the zona incerta brain expression seen previously in Ple232 KI mice [22], yet retained muscle specificity (Additional file 1: Figure S3). This serves as an example where moving the promoter to virus and/or minor base-pair modification altered the function of the promoter in one cell type, but not another. Second, when we tested hs671 and hs1218 each linked to the Hsp 68 mouse basal promoter as they were in the VISTA Enhancer project [38], we did not observe the expected regional specificity to either the forebrain or midbrain respectively (Additional file 1: Figure S4). Expression was noted in those regions, but similar expression was also detected throughout most other brain regions. Thus, we deemed these two constructs as being non-specific in our system. We hypothesize that these VISTA enhancers may only restrict expression embryonically, and not postnatally. We also tested from the literature PRS2/3:Con, a $D B H$-based promoter that was successfully used in adenovirus in the 

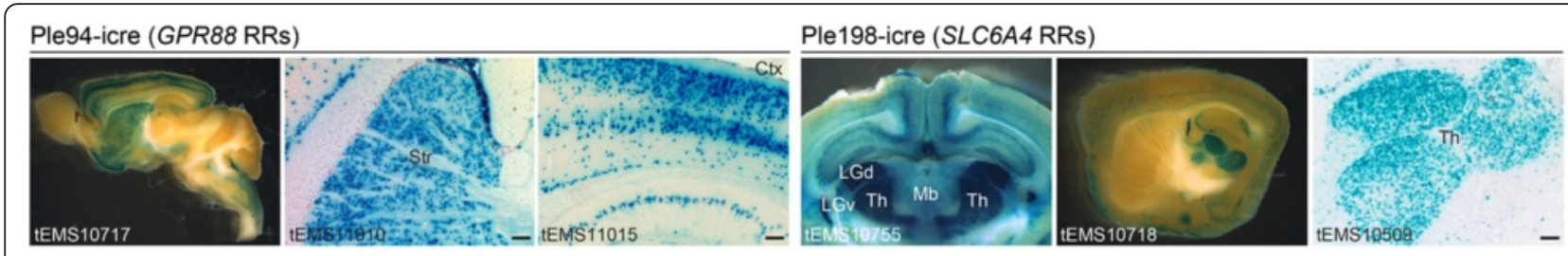

Ple251-icre (C8ORF46 RRs)
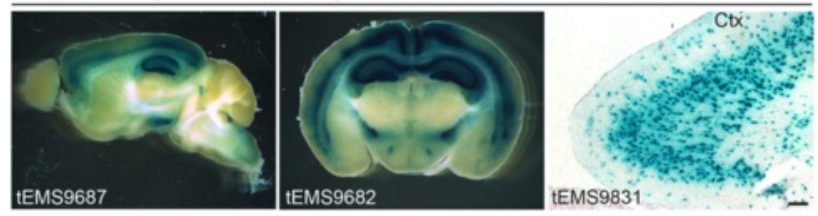

Ple251-icre-WPRE (C8ORF46 RRs)

Ple266-icre (S100B RRs)
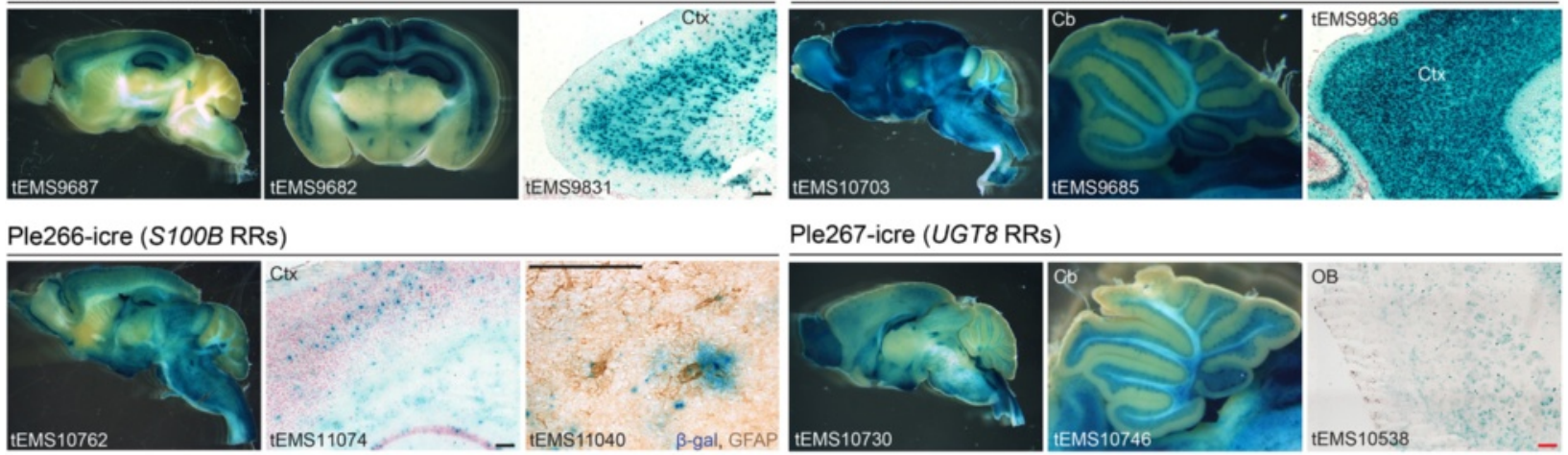

Ple267-icre (UGT8 RRs)

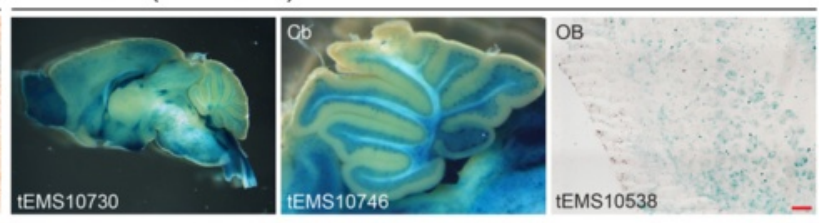

Ple303-icre (NOV RRs)
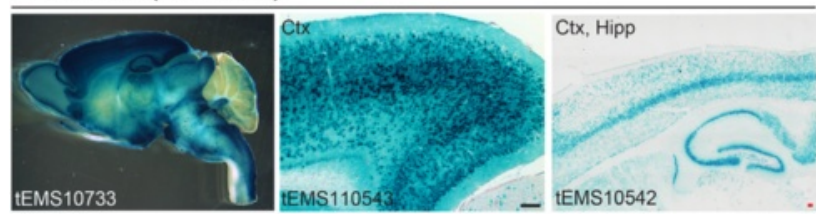

Ple304-icre (OLIG1 RRs)

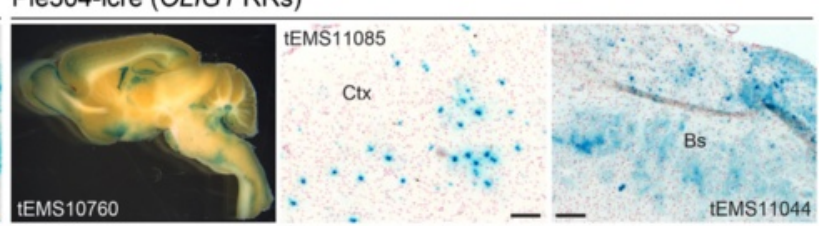

\section{Ple305-icre (OLIG1 RRs)}

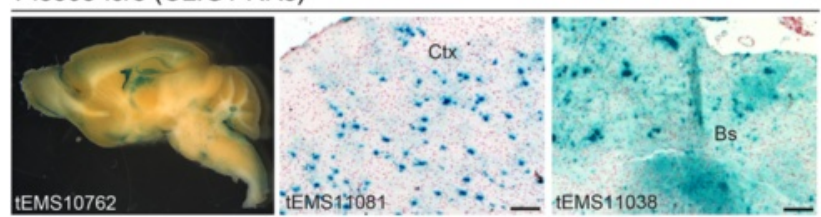

Fig. 3 Eight MiniPromoters in rAAVs show unique expression related to their source gene when driving icre in mice carrying a "Iox-stop-loxlacZ" allele, an historical-indirect reporter. Each tEMS number indicates an individual animal. Ple94-icre (GPR88 RRs) expressed strongly in the striatum and in upper cortical layers. Ple198-icre (SLC6A4 RRs) expressed in the thalamus and shows a clear demarcation between LGd and LGv. Ple251-icre (C8ORF46 RRs) demonstrated strong expression in the hippocampus and cortex; layers IV, V, and Vb are predominant. The zona incerta is also labeled. Ple251-icre-WPRE (C8ORF48 RRs) extended the expression to most brain regions, particularly throughout the cortex and in the cerebellar granular layer. Ple266-icre (S100B RRs) expressed sporadically in the brain with puffy processes in the cortex and corpus callosum. As expected for partial viral transduction, a subset of GFAP+ astrocytes are co-labelled with $\beta$-gal. Ple267-icre (UGT8 RRs) has strong staining in the olfactory bulb and in the cerebellar granule layer, with some Purkinje cells labeled. In the OB, globeruli-like structures are stained. Ple303-icre (NOV RRs) stains all cortical layers in midline and becomes specific to cortical layer V in more lateral sections. Ple304-icre and Ple305-icre (both OLIG1 RRs) demonstrated an indistinguishable expression pattern with scattered cells in several brain regions, including the cortex and brainstem, resembling puffy processes of oligodendrocytes. Bs, brainstem; Cb, cerebellum; Ctx, cortex; Hipp, hippocampus; icre, improved cre recombinase; LGd, dorsal lateral geniculate; LGV, ventral lateral geniculate; Mb, midbrain; OB, olfactory bulb; RRs, regulatory regions; Str, striatum; Th, thalamus. Blue, $\beta$-gal positive; brown, DAB immunohistochemistry; red, neutral red. [Scale bars $=100 \mu$ m]

rat brain [39]. However, we found that with intravenous P0 delivery using rAAV it showed no expression different from background. We hypothesize that this difference may have been due to the developmental stage of delivery (P0 versus adult), the virus utilized, or the use of human sequence in mouse versus rat.

In the second screening step, representative successful promoters from step one were recloned driving the direct reporter EmGFP. Figure 4 demonstrates that the Ple67 (FEV RRs) and Ple155 (PCP2 RRs) MiniPs drive highly specific and expected expression when injected at P4 in the temporal vein. Additional file 1: Figure S5 presents additional supportive data for both these MiniPs injected at P0. Ple67 was derived from $F E V$, a gene expressed in the serotonergic neurons of the raphe nuclei in the brain [50]. Previously, Ple67-EGFP and -lacZ KI mice gave the expected raphe expression [22, 24]. Here, using rAAV, Ple67 again localized expression to the raphe, which was further enhanced with the WPRE (Fig. 4 and Additional file 1: Figure S5). Ple155 was 


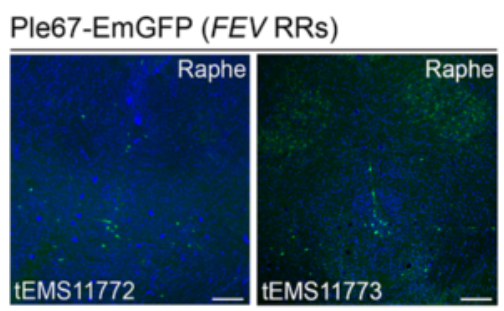

Ple67-EmGFP-WPRE (FEV RRs)
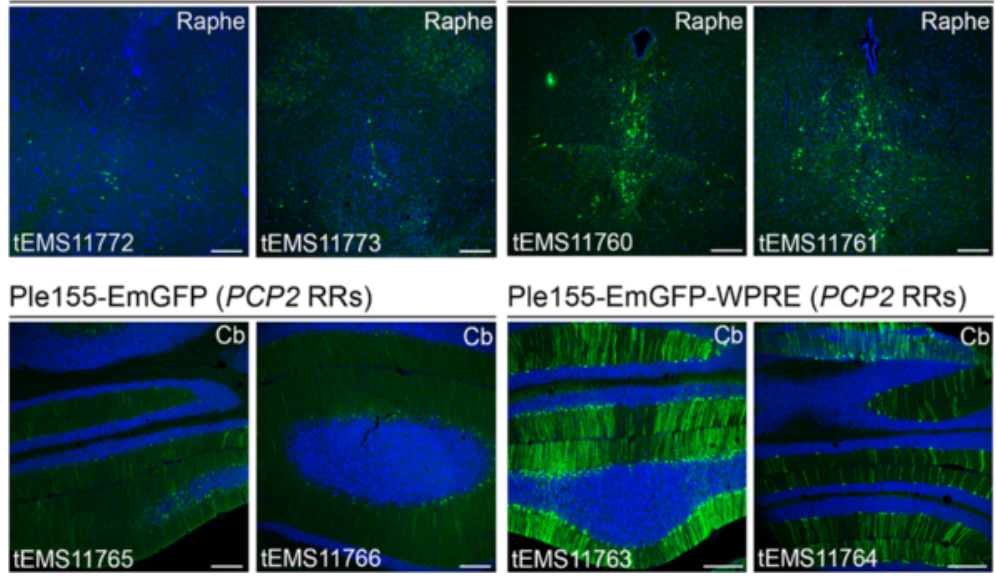

Ple155-EmGFP-WPRE (PCP2 RRs)

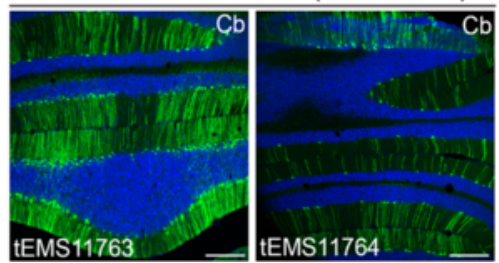

Fig. 4 Two MiniPromoters selected based on icre expression, recapitulated their unique expression related to their source gene when tested driving EmGFP. Ple67-EmGFP \pm WPRE (FEV RRs) drove expression in the dorsal raphe nuclei of the mouse brain. Ple155-EmGFP \pm WPRE (PCP2 RRs) drove expression in the Purkinje cells of the cerebellum, similar to the endogenous gene. In both cases, the presence of WPRE enhanced the number of cells and intensity of cells showing expression. Cb, cerebellum; EmGFP, emerald green fluorescent protein; Raphe, raphe nuclei; RRs, regulatory regions; WPRE, woodchuck hepatitis virus post-transcriptional regulatory element. Blue, Hoechst 33342; Green, EmGFP immunofluorescence.

[Scale bars $=200 \mu \mathrm{m}$ ]

derived from $P C P 2$, a gene expressed in the Purkinje cells of the cerebellum [51, 52]. However, Ple155-lacZ KI mice did not show this expected expression [22]. Thus, it was surprising when using rAAV, Ple155 gave strong and consistent expression in the Purkinje cells and their processes, which was further enhanced with the WPRE (Fig. 4, Additional file 1: Figure S5). Since expression with the ubiquitous promoters, even when enhanced with WPRE, was limited in Purkinje cells (Additional file 1: Figure S1), this result further expands the characterization of the viral tropism in this system. This result also suggests that KI at the Hprt genomic location is not permissive for $P C P 2$-based expression in Purkinje cells, or that the presumed multi-copy expression of virus in Purkinje cells may push the reporter above detection levels compared to the KI mouse. As anticipated, negative MiniP results were also obtained. Additional file 1: Figure S5 shows Ple264, which lacks elements 12 and 13 compared to the original Ple140 (NR2E1 RRs). While Ple140 was strongly positive in the hypothalamus of adult and embryonic KI mice [22, 24], Ple264 lost the hypothalamic expression when delivered in rAAV. This was presumably attributable to either the "cut down" or the viral delivery system.

Figure 5 presents two examples where the MiniP has been taken beyond screening, to modeling delivery applicable to gene therapy. For this, we evaluated the ability of Ple34 and Ple261 (CLDN5 RRs) to drive expression in the endothelial cells of the blood brain barrier, an important therapeutic target. Ple34 was previously positive driving lacZ in a KI mouse [24], and the "cut down" version, Ple261 (Fig. 2), was tested driving icre by temporal vein injection at P0. However, tail vein injection in adult mice models a possible therapeutic delivery, and allows evaluation of the expression with a mature blood-brain barrier. Figure $5 \mathrm{a}$ and $\mathrm{b}$ respectively, show consistent overlap of Ple34-miniSOG (a fluorescent reporter chosen for its small size [53]) and Ple261-EGFP, with the endothelial cell marker CD31. Importantly, MiniP-driven miniSOG or EGFP co-labeling with endothelial markers in the liver (CD16/CD32) and heart (CD31) was absent.

Overall, this data demonstrates that many of the Pleiades MiniPs expressed in rAAV according to their design source and thus may be useful for rAAV-mediated "gene-of-interest" delivery in the brain.

\section{MiniPromoters for the eye include restricted expression in bipolar ON cells and Müller glia}

Due to developmental similarities, we anticipated that many of the MiniPs designed for use in the brain would also be applicable to basic, preclinical, and even clinical use in ocular gene therapy. Thus, all 19 Pleiades MiniPs tested in rAAV2/9 were examined for expression in the eye after intravenous injection into neonatal mice. As in the brain, the historical-indirect icre reporter system gave a generalized pattern of positive staining that was sparse but widespread. However, in some cases superimposed on this was the expected restricted expression. As with the brain, only the GFP data is presented for MiniPs selected for second-step screening to avoid duplication. In all, we obtained restricted expression in 10 MiniPs in the eye. 
A

Ple34-miniSOG (CLDN5 RRs; 3,854 bp)
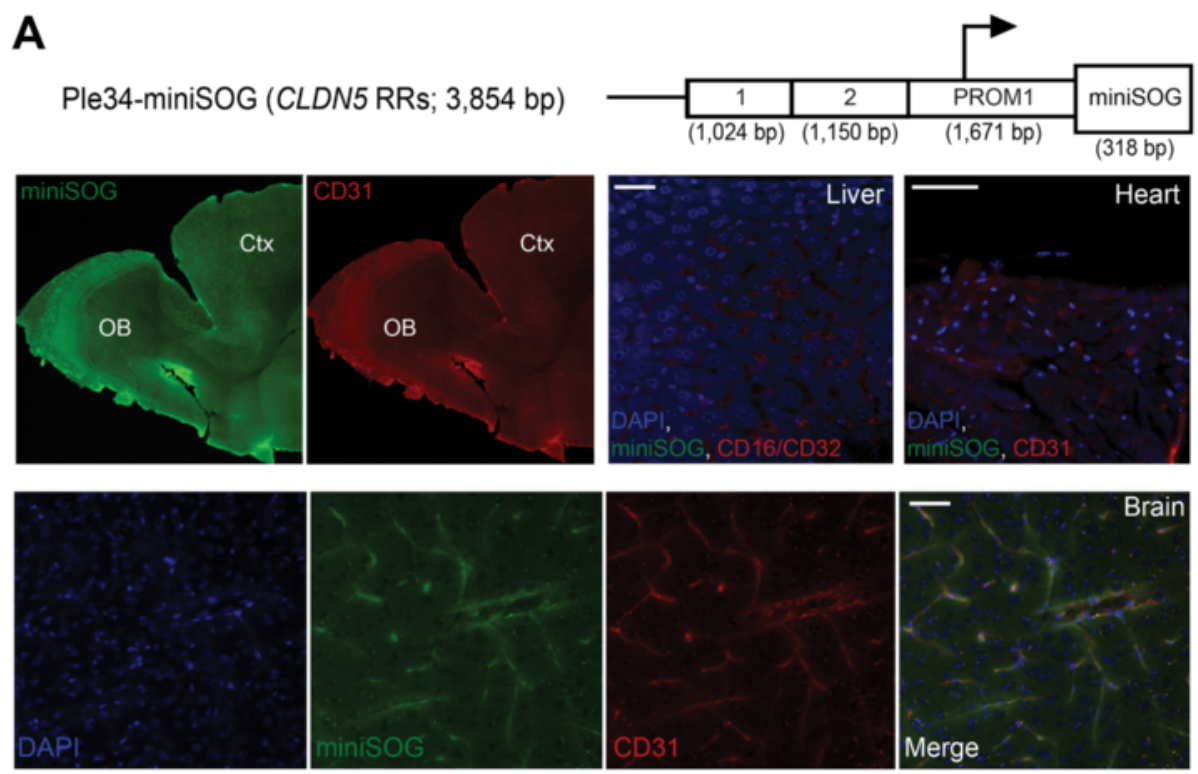

B

Ple261-EGFP (CLDN5 RRs; 2,963 bp)
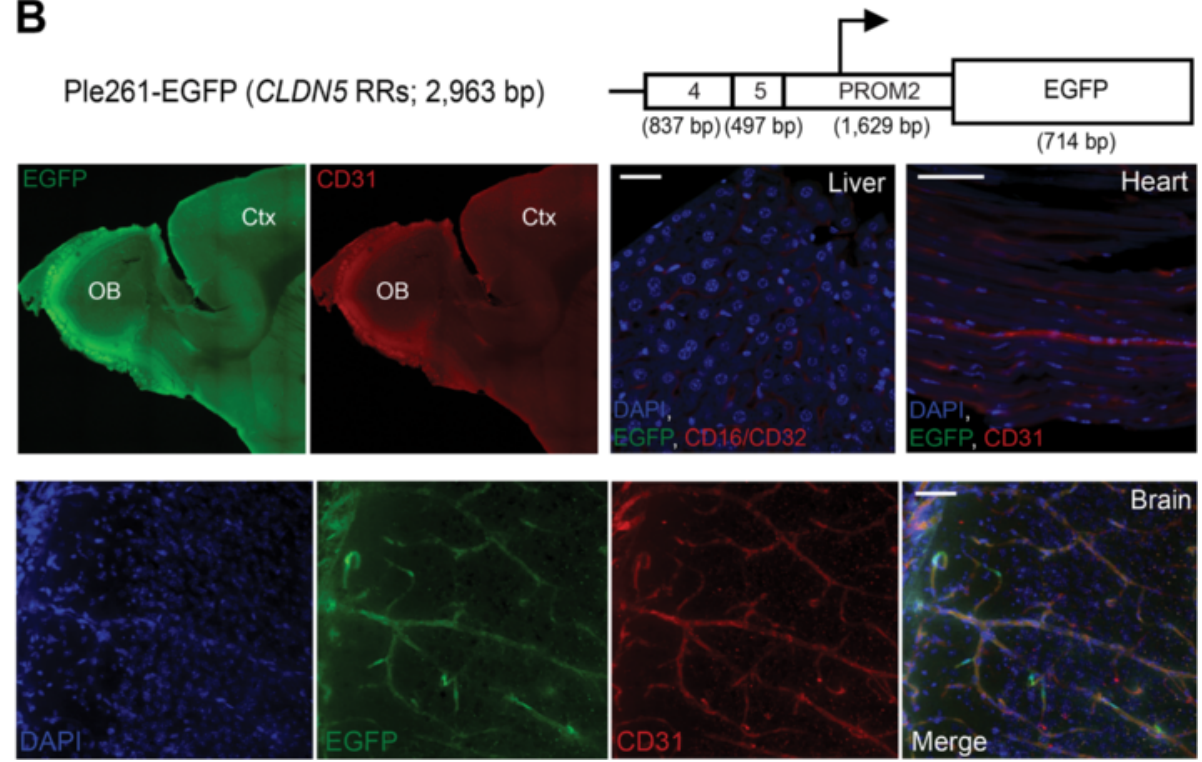

Fig. 5 Two CLDN5 RRs-based MiniPromoters (MiniPs) showed brain endothelial cell-specific expression related to their source gene, when virus was introduced into the adult circulatory system. a The Ple34 MiniP drove expression of the miniSOG fluorescent green reporter in blood vessels in the brain. Co-labelling was observed with CD31, a marker of endothelial cells. No miniSOG expression was detected in the heart where CD31 stains endocardial endothelium, or liver, where CD16/32 stains endothelial cells. b Ple261, a "cut down" of Ple34, also drove expression of EGFP in blood vessels in the brain. Again, co-labelling was observed with CD31, a marker of endothelial cells, but no expression was detected in the heart or liver. bp, base pairs; Ctx, cortex; EGFP, enhanced green fluorescent protein; miniSOG, mini Singlet Oxygen Generator; OB, olfactory bulb; RRs, regulatory regions. [Scale bars $=50 \mu \mathrm{m}$ ]

Figure 6a presents the data for three positive Pleiades MiniPs driving the historical-indirect reporter icre in the retina of "lox-stop-lox-lacZ" mice after injection at P0. Ple266 (S100B RRs) stained rare Müller glia (one of two mice) above the background. Ple302 (DCX RRs) intensely stained the retinal ganglion cell layer (GCL). Ple302 is a "cut down" of Ple53 (Fig. 2), which in a KI mouse showed expression in the GCL, but also neurogenic regions of the brain [22, 24]. However, when Ple302 was used in rAAV, the restricted brain expression was lost, either due to the "cut down" or the viral delivery system. Notable is that the GCL is not a region of endogenous $D C X$ expression, but has been previously observed with another promoter similar to Ple53 [54]. Finally, Ple303 (NOV RRs) showed some modest enrichment in horizontal cells. 


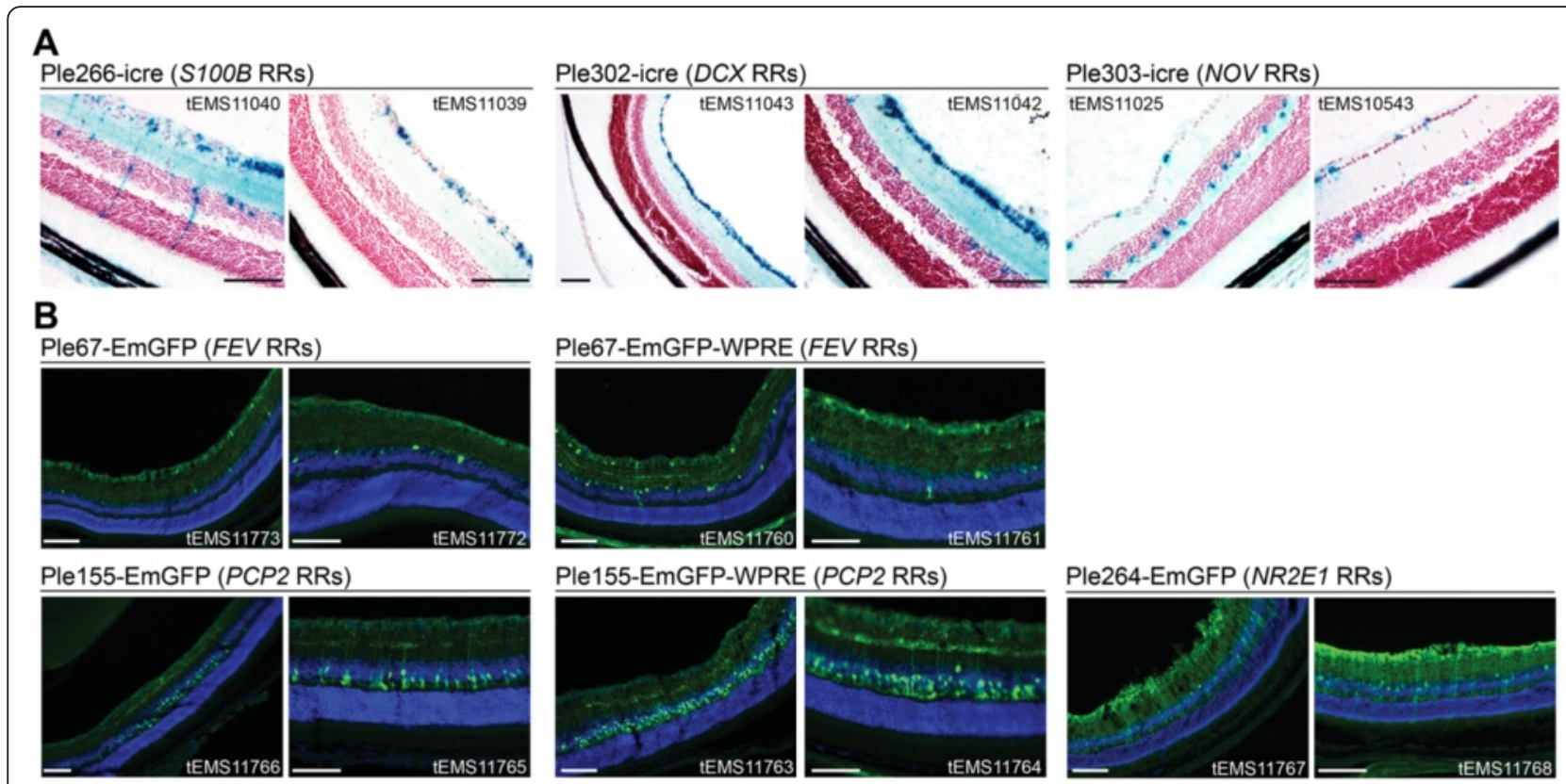

Fig. 6 Six MiniPromoters (MiniPs) showed cell type-specific expression in the retina. a The icre reporter led to identification of three retina positive MiniPs. Ple266-icre (S100B RRs) showed rare Müller glia expression as expected for the endogenous gene in one of two mice tested [69]. The astrocytic layer also demonstrated some staining. Ple302-icre (DCX RRs) intensely stained the ganglion cell layer (GCL), similar to previous DCXbased MiniPs [22]. For Ple303-icre (NOV RRs) we observed modest enrichment of staining in the outer side of the inner nuclear layer indicative of horizontal cells. Blue, $\beta$-gal positive; red, neutral red. b Constructs utilizing the EmGFP reporter identified an additional three MiniPs with retinal expression. In both cases tested, the presence of the WPRE enhanced the number and intensity of cells showing expression. Ple67-EmGFP \pm WPRE (FEV RRs) demonstrated expression primarily in the GCL and inner nuclear layer in putative ganglion and amacrine cells. Rarely, additional cells were also positive with the WPRE-containing virus. Ple155-EmGFP \pm WPRE (PCP2 RRs) restricted expression to bipolar ON cells. Ple264-EmGFP (NR2E1 RRs) showed strong staining of Müller glial cells. EmGFP, emerald green fluorescent protein; GCL, retinal ganglion cell layer; icre, improved cre recombinase; RRs, regulatory regions. [Scale bars $=100 \mu \mathrm{m}$ ]

In the second screening step, MiniPs driving the direct reporter EmGFP were positive in the retina and cornea after injection at P4. In Fig. $6 \mathrm{~b}$ retinal expression is presented. Ple67 (FEV RRs), showed expression primarily in the GCL and inner nuclear layer, indicative of putative ganglion and amacrine cells, which was enhanced by the presence of the WPRE. Ple155 (PCP2 RRs) showed restricted expression in bipolar ON cells. In this case, the presence of the WPRE allowed enhanced visualization of the cellular end feet. Note, comparison with previous work demonstrates that the expression of Ple67 and Ple155 is serotype and delivery-method independent [22, 55]. Finally, Ple264 (NR2E1 RRs) gave strong Müller glia staining, another gene therapy target [56]. Interestingly, Ple264 is a "cut down" of Ple140 which in KI mice expressed strongly in the hypothalamus, but was completely negative in the eye [22]. In Ple264, after the removal of elements 12 and 13, the brain expression was lost, either due to the "cut down" or viral delivery. However, Ple264 gained the NR2E1-endogenous expression in Müller glia [57, 58], either due to removal of inhibitory sequences from the promoter, the multi-copy rAAV system pushing the reporter above detection levels compared to the KI mouse, or non-permissive expression conditions at the Hprt genomic location for NR2E1-based expression in the KI mouse.

In the cornea (Additional file 1: Figure S6), we observed expression for five MiniPs driving icre (Ple251 (C8ORF46 RRs), Ple253 (PITX3 RR), hs671, Ple302 (DCX RRs), and Ple303 (NOV RRs), and one driving EmGFP (Ple67 (FEV RRs)). Typically, expression was stromal, but Ple251 also showed expression in the epithelial layer of the cornea. In the case of Ple251-icre, Ple253-icre, and Ple67-EmGFP, we were unable to detect corneal expression without the use of the WPRE.

Overall, this data represents a rich resource of therapeutically-enabling eye MiniPs that are likely to have efficacy similar to the preclinical use of the Ple155 MiniP, which has already been used to restore vision in a mouse model of congenital night blindness [55].

We have demonstrated that a single-step rAAV pipeline utilizing a direct reporter is sufficient for MiniP development. We initially established a two-step screening system, assuming we would need the sensitivity of a historical-indirect reporter system to detect expression from restricted promoters. However, we have found there are disadvantages to such a sensitive system, and that detection of the direct EmGFP reporter was not 
limiting, perhaps because of the multi-copy rAAV system, although occasionally requiring anti-GFP immunohistochemistry or a WPRE for increased expression. Importantly for this approach, we showed that the positive impact of WPRE was independent of MiniP, or open reading frame, and that in the two cases for which cell specificity could be best determined (Ple67-EmGFP and Ple155-EmGFP (Figs. 4, 6)), expression in the brain and retina was not "broadened" by WPRE. Given this data, we suggest utilization of a single-step direct reporter and the WPRE in future work.

The 16 MiniPs (human DNA elements designed to drive expression in restricted cell types) presented here are important advances for basic and preclinical research, and may enable a paradigm shift in clinical gene therapy. To assess the effectiveness of moving MiniPs from KI mice to rAAV, we utilized five previous Pleiades designs and $100 \%$ showed expression related to the gene and source. This demonstrates that the previously published Pleiades MiniPs provide a rich resource of constructs for use in rAAV. However, many previous MiniPs will still need to be "cut-down" for use in rAAV. Thus, we undertook this work for eight Pleiades MiniPs, of which five demonstrated expression related to their gene and source, and two MiniPs maintained a further restricted aspect of their previous expression. Therefore, $87.5 \%$ maintained similar expression patterns despite extensive size reduction. In addition, for one gene we attempted, and successfully developed, a new promoter rapidly and inexpensively. Finally, we demonstrate the ability to test MiniPs directly in a single step in the mouse EmGFP viral pipeline. Therefore, we provide extensive evidence for the utilization of a high-throughput method of promoter design and testing in rAAV. These MiniPs are now available unrestricted to the academic community.

\section{Methods}

\section{MiniPromoter bioinformatics and design}

MiniPromoter (MiniP) bioinformatics has been described in part, previously [24]. For our cut-downs and new designs we considered the UCSC genome browser [59] tracks: ENCODE datasets [60-66], TFBS conserved, VISTA enhancers [38], UCSF brain methylation, ORegAnno [67, 68], CpG islands, human mRNAs/ESTs, CD34 DNAse I, Repeat Masker, and Conservation. For the Conservation tracks, we utilized the mammalian conservation and Multiz alignments including placental mammals and vertebrates, but excluded non-human primates to minimize selection bias that may be introduced by high sequence similarities between humans and primates. The following histone modifications were considered: methylation of H3K4 and H3K36 as indicators of transcriptional activation, methylation of H3K9 and H3K27 for transcriptional silencing, and acetylation of H3K4 and H3K27 indicating transcriptional activation. Tracks such as TFBS conserved, VISTA enhancers, and ORegAnno provide in silico and/or experimental evidence for transcription factor binding sites and regulatory regions. Tracks for DNAse I hypersensitivity, such as CD34 DNAse I, help to mark regions that tend to be associated with open chromatin structure and may contain active binding sites. Potential regulatory regions were rated as containing 1 to $5+$ features; the highest scores typically being chosen for testing in MiniPs.

\section{Mice}

We purchased heterozygous 129S-Gt(ROSA)26Sor ${ }^{\text {tm } 1 S o r} /$ J mice at N? + 9p (JAX Stock No: 003310) and continued backcrossing the allele onto the 129S1/SvImJ strain (JAX Stock No: 002448) until N13. Heterozygous mice were then intercrossed to obtain a congenic homozygous strain. We also purchased homozygous B6.129S4-Gt(ROSA)26Sor ${ }^{\text {tm1Sor }} / \mathrm{J}$ mice at N4F10N1p (JAX Stock No: 003474) and restarted backcrossing the allele onto the C57BL/6J strain (JAX Stock No: 000664) until N11. Heterozygous mice were then intercrossed to obtain a congenic homozygous strain. All experimental mice were B6129F1 hybrids and generated either as the first generation of crossing a congenic homozygous 129S-Gt(ROSA)26Sor ${ }^{\text {tm1Sor }} / \mathrm{JEms}$ to a congenic homozygous B6.129S4-Gt(ROSA)26Sor ${ }^{\text {tmisor } / J E m s, ~ o r ~ C 57 B L / 6 J ~ t o ~}$ $129 \mathrm{~S} 1 /$ SvImJ. For clarity, we describe mice carrying the Gt(ROSA)25Sor ${ }^{\text {tm ISor }}$ allele as containing a "lox-stop-loxlacZ" reporter, which we utilized as an indirect-historical marker of cre-recombinase expression.

\section{Virus generation and analysis Virus production}

We generated a "plug and play" rAAV2 genome plasmid that included AAV2 inverted terminal repeats (ITR), and allowed for the easy exchange of promoters, intron, reporters, and 3' UTR elements using restriction enzymes. In this study we tested the 3' UTR element WPRE (woodchuck hepatitis virus post-transcriptional regulatory element) mut6 [32, 33]. MiniPs were isolated from existing Pleiades Promoter plasmids [22, 24], or generated by direct synthesis (DNA2.0, Menlo Park, CA, USA), and cloned into the multiple-cloning site (MCS) of a new "plug and play" rAAV2 genome plasmid - either the pEMS1987 backbone containing the improved cre (icre) reporter [28], and/or the pEMS2157 backbone containing the emerald green fluorescent protein (EmGFP) reporter [29]. Plasmids were propagated in the E. coli SURE Cells (Agilent Technologies, Santa Clara, CA, USA). One to five $\mu \mathrm{g}$ of plasmid DNA containing the MiniP was prepared by QIAgen Spin MiniPrep Kit (Catalog \#27104, QIAgen, Germantown, MD, USA). rAAV plasmid DNAs was demonstrated free of rearrangements by $A h d \mathrm{I}$ digest, ITRs 
verified via SmaI single digests, and overall structure via AscI/EcoRI double digestion, and upon confirmation, sent to the Vector Core at the University of Pennsylvania (Philadelphia, PA, USA) for large-scale DNA amplification using the EndoFree Plasmid Mega Kit (Catalog \#21381, QIAgen, Germantown, MD, USA). Quality control on the plasmid preparation was done via SmaI, PvuII, and SnaBI confirmatory digests, and subsequently packaged into rAAV2/9 serotype virus. Ple34-miniSOG and Ple261EGFP were cloned, DNA amplified, and packaged into AAV2/9 in the laboratory of A.A. for adult intravenous experiments.

\section{Virus injection}

B6129F1 hybrid pups were either homozygous for the Gt(ROSA)26Sor ${ }^{\text {tm1Sor }}$ allele for icre virus injections, or wild type at that locus for EmGFP virus injections. Timed pregnancies were achieved using crowded females, experienced studs, and plug checking of females such that the day of birth could be accurately predicted. Virus injections were into postnatal (P) 0 (the day of birth) pups for icre constructs, and P4 for EmGFP constructs. For P0, if the female gave birth over night or in the morning, virus was injected in the afternoon. If she gave birth in the afternoon, virus was injected the next morning. Standard P0 and P4 pup injections were into the superficial temporal vein using $1 \times 10^{13} \mathrm{GC} / \mathrm{mL}$ (genome copies per milliliter) virus in a volume of $50 \mu \mathrm{L}$ (in PBS) with a 30-gauge needle and a 1 cc syringe. After injection, pups were tattooed for identification and returned to their cage. Ple34-miniSOG (dose of $3 \times 10^{11}$ viral genomes) and Ple261-EGFP (dose of $8 \times 10^{11}$ viral genomes) virus were injected intravenously at $6-8$ weeks via tail vein.

\section{Harvesting of animals}

Virus-injected mice were harvested at P21 or P56. Animals were given a lethal dose of avertin injected intraperitoneally. Thereafter, perfusion with 1xPBS for 2 min and $4 \%$ PFA/PBS for $8 \mathrm{~min}$ was performed. Brain, eye, spinal cord, and heart were harvested and post-fixed for $2 \mathrm{~h}$ at $4{ }^{\circ} \mathrm{C}$. The tissues were then stored in $0.01 \%$ azide/PBS at $4{ }^{\circ} \mathrm{C}$. Ple34-miniSOG and Ple261-EGFP mice were harvested 4 weeks post injection.

\section{Histology}

Reporter gene expression was usually analyzed at P21 or P56 in brain, eye, spinal cord, and heart. Tissues were cryoprotected in $25 \%$ sucrose/PBS overnight at $4{ }^{\circ} \mathrm{C}$. After embedment in OCT (optimal cutting temperature compound) the following day, $20 \mu \mathrm{m}$ sections were directly mounted onto slides. For X-gal (5-bromo-4chloro-3-indolyl- $\beta$-D-galacto-pyranoside) staining, tissues were rinsed in PBS and Triton-X/PBS and stained in $0.1 \%$ X-gal solution overnight $(\sim 18 \mathrm{~h})$ at $37{ }^{\circ} \mathrm{C}$. After staining, sections were rinsed and counterstained with neutral red, dehydrated, and mounted with coverslips. For co-labeling of X-gal with markers using immunohistochemistry, standard procedures were followed and the X-gal stain was performed either prior to primary antibody incubation or between primary and secondary antibodies, depending on the strength of the $\mathrm{X}$-gal stain. X-gal stains blue any cells that have recombined the "lox-stop-lox-lacZ" reporter locus due to icre recombinase activity and thus expressing the $\beta$-galactosidase protein. With the icre reporter, we detected historical and indirect expression of the promoter.

EmGFP constructs were signal amplified using a chicken $\alpha$-GFP antibody (Aves Labs Inc.; 1:500) and Alexa Fluor 488 secondary (Life Technologies; 1:1000). With the EmGFP fluorescent reporter, we visualized direct expression. Ple34-miniSOG and Ple261-EGFP mice were analyzed for green epifluorescence (miniSOG or EGFP) and co-stained with markers of endothelial cells (brain and heart: CD31; liver: CD16/CD32).

\section{Imaging}

Between four and twelve animals were studied for each MiniPromoter, and unique expression patterns of the MiniPs were determined by microscope and image analyses. The images shown are typically from mice that had the most successful injections and thus the full dose of rAAV, and therefore the greatest likelihood to observe off-target expression if present. Brightfield and fluorescence images were standardly taken using an Olympus BX61 motorized microscope or a Zeiss 710 Confocal Laser Scanning Microscope. Images were processed using ImageJ (http://rsbweb.nih.gov/ij/), Photoshop, and Illustrator (Adobe, San Jose, CA). Brightness, contrast, and color balancing adjustments, as per Molecular Brain guidelines, were made where necessary to improve visibility.

\section{Statement of ethics approval}

All procedures involving animals were in accordance with the Canadian Council on Animal Care and University of British Columbia Animal Care Committee (protocols \#A10-0268, and A10-0269). All animal experiments were carried out in accordance to NIH guidelines and as approved by the UNC Institutional Animal Care and Use Committee (IACUC).

\section{Statement of consent for publication}

Not applicable. 


\section{Materials and data availability}

All MiniPs and constructs have been deposited with AddGene (www.addgene.org), with the exception of those constructs used in generating Fig. 5 - these are available from A.A. upon request (aravind_asokan@med.unc.edu).

\section{Additional file}

Additional file 1: Supplementary Figures 1 - 6. (PDF 10 kb)

\section{Abbreviations}

BAC: bacterial artificial chromosome; bp: base pairs; Bs: brainstem; Cb: cerebellum; Ctx: cortex; EGFP: enhanced green fluorescent protein; EmGFP: emerald green fluorescent protein; GCL: retinal ganglion cell layer; Hipp: hippocampus; icre: improved cre recombinase; ITR: inverted terminal repeat; Kl: knock-in; LGd: dorsal lateral geniculate; LGv: ventral lateral geniculate; Mb: midbrain; MCS: multiple cloning site; MiniP: MiniPromoter; miniSOG: mini Singlet Oxygen Generator; NA: not applicable; OB: olfactory bulb; ORF: open reading frame; PMID: PubMed ID number; rAAV: recombinant adeno associated virus; Raphe: raphe nuclei; RRs: regulatory regions; ST: stereotaxic; Str: striatum; Th: thalamus; VISTA RI: random insertion from VISTA enhancer project; WPRE: woodchuck hepatitis virus post-transcriptional regulatory element.

\section{Competing interests}

CNdL, VC, CAD, EP-C, SJMJ, RAH, DG, WWW, EMS, and the University of British Columbia have filed for US patents on a subset of the MiniPs. The authors declare that they have no competing interests.

\section{Authors' contributions}

CNdL and EMS analyzed the data and wrote the manuscript. EMS. conceived the study. SJMJ, RAH, DG, WWW, and EMS supervised the study. CNdL, AJK, GEB, JWH, SLL, TCL, RJB, LJB, VC, AYC, CAD, OK, SL, SCM, EP-C, MIS-N, KW, GSY, and MZ performed experiments and collected data. All authors read and approved the final manuscript.

\section{Acknowledgments}

We thank: Johar Ali and Behzad Imanian for primer design; Kathleen G. Banks, Sonia F. Black, and Tom W. Johnson for viral injection groundwork; and Sujin Im and Clara Van Ommen for histological support.

\section{Funding}

This work was supported in main by Genome British Columbia grant AGCPCanEuCre-01, and in part by: Genome Canada; GlaxoSmithKline R\&D Ltd.; BC Mental Health and Addiction Services; Child and Family Research Institute; University of British Columbia (UBC) Institute of Mental Health; UBC Office of the Vice President Research; NIH National Heart, Lung, and Blood Institute; Brain Canada; and the Quebec Consortium for Drug Discovery.

\section{Author details}

${ }^{1}$ Centre for Molecular Medicine and Therapeutics at the Child \& Family Research Institute, University of British Columbia, 950 W 28 Ave, Vancouver, BC V5Z 4H4, Canada. '2Department of Medical Genetics, University of British Columbia, Vancouver, BC V6H 3N1, Canada. ${ }^{3}$ Gene Therapy Centre, University of North Carolina, Chapel Hill, NC 27599, U.S.A.. ${ }^{4}$ Canada's Michael Smith Genome Sciences Centre, British Columbia Cancer Agency, Vancouver, BC V5Z 4S6, Canada. ${ }^{5}$ Department of Molecular Biology and Biochemistry, Simon Fraser University, Burnaby, BC V5A 156, Canada. ${ }^{6}$ Department of Psychiatry, University of British Columbia, Vancouver, BC V6T 2A1, Canada.

Received: 21 January 2016 Accepted: 30 April 2016 Published online: 10 May 2016

\section{References}

1. Bradley A, Anastassiadis K, Ayadi A, Battey J, Bell C, Birling M, Bottomley J, Brown S, Bürger A, Bult C, et al. The mammalian gene function resource: the international knockout mouse consortium. Mamm Genome. 2012;23(9-10): 580-6.
2. Schmouth JF, Castellarin M, Laprise S, Banks KG, Bonaguro RJ, Mclnerny SC, Borretta L, Amirabbasi M, Korecki AJ, Portales-Casamar E, et al. Non-codingregulatory regions of human brain genes delineated by $B A C$ knock-in mice. BMC Biol. 2013;11(1):106.

3. Murray SA, Eppig JT, Smedley D, Simpson EM, Rosenthal N. Beyond knockouts: cre resources for conditional mutagenesis. Mamm Genome. 2012;23(9-10):587-99.

4. Gompf HS, Budygin EA, Fuller PM, Bass CE. Targeted genetic manipulations of neuronal subtypes using promoter-specific combinatorial AAVs in wildtype animals. Front Behav Neurosci. 2015;9:152.

5. Perea G, Yang A, Boyden ES, Sur M. Optogenetic astrocyte activation modulates response selectivity of visual cortex neurons in vivo. Nat Commun. 2014:5:3262

6. Deng WT, Dyka FM, Dinculescu A, Li J, Zhu P, Chiodo V, Boye SL, Conlon TJ, Erger KE, Cossette T, et al. Stability and Safety of an AAV Vector for Treating RPGR-ORF15 X-linked Retinitis Pigmentosa. Hum Gene Ther. 2015;26(9):593-602.

7. Naldini L. Gene therapy returns to centre stage. Nature. 2015;526(7573):351-60.

8. Maguire CA, Ramirez SH, Merkel SF, Sena-Esteves M, Breakefield XO. Gene Therapy for the Nervous System: Challenges and New Strategies. Neurotherapeutics. 2014;11(4):817-39.

9. Naidoo J, Young D. Gene regulation systems for gene therapy applications in the central nervous system. Neurol Res Int. 2012;2012:595410.

10. Kugler S. Tissue-Specific Promoters in the CNS. Methods Mol Biol. 2016; 1382:81-91

11. Mingozzi F, High KA. Therapeutic in vivo gene transfer for genetic disease using AAV: progress and challenges. Nat Rev Genet. 2011;12(5):341-55.

12. Vandenberghe $\mathrm{LH}$, Auricchio A. Novel adeno-associated viral vectors for retinal gene therapy. Gene Ther. 2012;19(2):162-8.

13. Askou AL. Development of gene therapy for treatment of age-related macular degeneration. Acta Ophthalmol. 2014;92(Suppl Thesis3):1-38.

14. Hufnagel RB, Ahmed ZM, Correa ZM, Sisk RA. Gene therapy for Leber congenital amaurosis: advances and future directions. Graefes Arch Clin Exp Ophthalmol. 2012;250(8):1117-28.

15. Jacobson SG, Cideciyan AV, Ratnakaram R, Heon E, Schwartz SB, Roman AJ, Peden MC, Aleman TS, Boye SL, Sumaroka A, et al. Gene therapy for leber congenital amaurosis caused by RPE65 mutations: safety and efficacy in 15 children and adults followed up to 3 years. Arch Ophthalmol. 2012;130(1):9-24.

16. Maclaren RE, Groppe M, Barnard AR, Cottriall CL, Tolmachova T, Seymour L, Clark KR, During MJ, Cremers FP, Black GC, et al. Retinal gene therapy in patients with choroideremia: initial findings from a phase 1/2 clinical trial. Lancet. 2014;383:1129-37.

17. Lentz TB, Gray SJ, Samulski RJ. Viral vectors for gene delivery to the central nervous system. Neurobiol Dis. 2012;48(2):179-88.

18. Dong JY, Fan PD, Frizzell RA. Quantitative analysis of the packaging capacity of recombinant adeno-associated virus. Hum Gene Ther. 1996;7(17):2101-12.

19. Green ED, Guyer MS. Charting a course for genomic medicine from base pairs to bedside. Nature. 2011;470(7333):204-13.

20. O'Connor TR, Bailey TL. Creating and validating cis-regulatory maps of tissuespecific gene expression regulation. Nucleic Acids Res. 2014;42(17):11000-10.

21. Girgis HZ, Ovcharenko I. Predicting tissue specific cis-regulatory modules in the human genome using pairs of co-occurring motifs. BMC Bioinformatics. 2012;13:25.

22. de Leeuw CN, Dyka FM, Boye SL, Laprise S, Zhou M, Chou AY, Borretta L', Mclnerny SC, Banks KG, Portales-Casamar E, et al. Targeted CNS delivery using human MiniPromoters and demonstrated compatibility with adenoassociated viral vectors. Mol Ther Methods Clin Dev. 2014;1(5):1-15.

23. Yang C, McLeod AJ, Cotton AM, de Leeuw CN, Laprise S, Banks KG, Simpson EM, Brown CJ. Targeting of $>1.5 \mathrm{Mb}$ of human DNA into the mouse $X$ chromosome reveals presence of cis-acting regulators of epigenetic silencing. Genetics. 2012;192(4):1281-93.

24. Portales-Casamar E, Swanson DJ, Liu L, de Leeuw CN, Banks KG, Ho Sui SJ, Fulton DL, Ali J, Amirabbasi M, Arenillas DJ, et al. A regulatory toolbox of MiniPromoters to drive selective expression in the brain. Proc Natl Acad Sci U S A. 2010;107(38):16589-94.

25. Yang GS, Banks KG, Bonaguro RJ, Wilson G, Dreolini L, de Leeuw CN, Liu L, Swanson DJ, Goldowitz D, Holt RA, et al. Next Generation Tools for Highthroughput Promoter and Expression Analysis Employing Single-copy Knock-ins at the Hprt1 Locus. Genomics. 2009:93:196-204.

26. D'Souza CA, Chopra V, Varhol R, Xie YY, Bohacec S, Zhao Y, Lee LL, Bilenky M, Portales-Casamar E, He A, et al. Identification of a set of genes showing regionally enriched expression in the mouse brain. BMC Neurosci. 2008;9:66. 
27. Foust KD, Nurre E, Montgomery CL, Hernandez A, Chan CM, Kaspar BK. Intravascular AAV9 preferentially targets neonatal neurons and adult astrocytes. Nat Biotechnol. 2009;27(1):59-65.

28. Shimshek DR, Kim J, Hubner MR, Spergel DJ, Buchholz F, Casanova E, Stewart AF, Seeburg PH, Sprengel R. Codon-improved Cre recombinase (iCre) expression in the mouse. Genesis. 2002;32(1):19-26.

29. Teerawanichpan P, Hoffman T, Ashe P, Datla R, Selvaraj G. Investigations of combinations of mutations in the jellyfish green fluorescent protein (GFP) that afford brighter fluorescence, and use of a version (VisGreen) in plant, bacterial, and animal cells. Biochim Biophys Acta. 2007;1770(9):1360-8.

30. de Hoon M, Shin JW, Carninci P. Paradigm shifts in genomics through the FANTOM projects. Mamm Genome. 2015;26(9-10):391-402.

31. Mathelier A, Zhao X, Zhang AW, Parcy F, Worsley-Hunt R, Arenillas DJ, Buchman S, Chen CY, Chou A, lenasescu H, et al. JASPAR 2014: an extensively expanded and updated open-access database of transcription factor binding profiles. Nucleic Acids Res. 2014;42(Database issue):D142-147.

32. Zufferey R, Donello JE, Trono D, Hope TJ. Woodchuck hepatitis virus posttranscriptional regulatory element enhances expression of transgenes delivered by retroviral vectors. J Virol. 1999;73(4):2886-92.

33. Zanta-Boussif MA, Charrier S, Brice-Ouzet A, Martin S, Opolon P, Thrasher AJ, Hope TJ, Galy A. Validation of a mutated PRE sequence allowing high and sustained transgene expression while abrogating WHV-X protein synthesis: application to the gene therapy of WAS. Gene Ther. 2009;16(5):605-19.

34. Byrne LC, Lin YJ, Lee T, Schaffer DV, Flannery JG. The expression pattern of systemically injected AAV9 in the developing mouse retina is determined by age. Mol Ther. 2014;23(2):290-6.

35. Lenhard B, Sandelin A, Carninci P. Metazoan promoters: emerging characteristics and insights into transcriptional regulation. Nat Rev Genet. 2012;13(4):233-45.

36. Juven-Gershon T, Hsu JY, Kadonaga JT. Perspectives on the RNA polymerase II core promoter. Biochem Soc Trans. 2006;34(Pt 6):1047-50.

37. Kadonaga JT. Perspectives on the RNA polymerase II core promoter. Wiley Interdiscip Rev Dev Biol. 2012;1(1):40-51

38. Visel A, Minovitsky S, Dubchak I, Pennacchio LA. VISTA Enhancer Browser-a database of tissue-specific human enhancers. Nucleic Acids Res. 2007; 35(Database issue):D88-92.

39. Hwang DY, Hwang MM, Kim HS, Kim KS. Genetically engineered dopamine beta-hydroxylase gene promoters with better PHOX2-binding sites drive significantly enhanced transgene expression in a noradrenergic cell-specific manner. Mol Ther. 2005;11(1):132-41.

40. Aschauer DF, Kreuz S, Rumpel S. Analysis of transduction efficiency, tropism and axonal transport of AAV serotypes 1, 2, 5, 6, 8 and 9 in the mouse brain. PLoS One. 2013;8(9):e76310.

41. Murlidharan G, Samulski RJ, Asokan A. Biology of adeno-associated viral vectors in the central nervous system. Front Mol Neurosci. 2014;7:76.

42. Watakabe A, Ohtsuka M, Kinoshita M, Takaji M, Isa K, Mizukami H, Ozawa K, Isa T, Yamamori T: Comparative analyses of adeno-associated viral vector serotypes 1, 2, 5, 8 and 9 in marmoset, mouse and macaque cerebral cortex. Neurosci Res. 2014;25240284.

43. Gray SJ, Foti SB, Schwartz JW, Bachaboina L, Taylor-Blake B, Coleman J, Ehlers MD, Zylka MJ, McCown TJ, Samulski RJ. Optimizing promoters for rAAVmediated gene expression in the peripheral and central nervous system using self-complementary vectors. Hum Gene Ther. 2011;22(9):1143-53.

44. Korecka JA, Schouten M, Eggers R, Ulusoy A, Bossers K, Verhaagen J: Comparison of AAV serotypes for gene delivery to dopaminergic neurons in the substantia nigra. In: Viral Gene Therapy. Edited by Xu K: InTech; 2011: 450

45. Ng L, Bernard A, Lau C, Overly CC, Dong HW, Kuan C, Pathak S, Sunkin SM, Dang C, Bohland JW, et al. An anatomic gene expression atlas of the adult mouse brain. Nat Neurosci. 2009;12(3):356-62.

46. Zhuang X, Masson J, Gingrich JA, Rayport S, Hen R. Targeted gene expression in dopamine and serotonin neurons of the mouse brain. $J$ Neurosci Methods. 2005;143(1):27-32.

47. Chang AS, Chang SM, Starnes DM, Schroeter S, Bauman AL, Blakely RD. Cloning and expression of the mouse serotonin transporter. Brain Res Mol Brain Res. 1996:43(1-2):185-92.

48. Lebrand C, Cases O, Wehrle R, Blakely RD, Edwards RH, Gaspar P. Transient developmental expression of monoamine transporters in the rodent forebrain. J Comp Neurol. 1998;401(4):506-24.

49. Lein ES, Hawrylycz MJ, Ao N, Ayres M, Bensinger A, Bernard A, Boe AF, Boguski MS, Brockway KS, Byrnes EJ, et al. Genome-wide atlas of gene expression in the adult mouse brain. Nature. 2007;445(7124):168-76.
50. Hendricks T, Francis N, Fyodorov D, Deneris ES. The ETS domain factor Pet-1 is an early and precise marker of central serotonin neurons and interacts with a conserved element in serotonergic genes. J Neurosci. 1999:19(23):10348-56.

51. Oberdick J, Smeyne RJ, Mann JR, Zackson S, Morgan JI. A promoter that drives transgene expression in cerebellar Purkinje and retinal bipolar neurons. Science. 1990;248(4952):223-6.

52. Vandaele S, Nordquist DT, Feddersen RM, Tretjakoff I, Peterson AC, Orr HT Purkinje cell protein-2 regulatory regions and transgene expression in cerebellar compartments. Genes Dev. 1991;5(7):1136-48.

53. Shu X, Lev-Ram V, Deerinck TJ, Qi Y, Ramko EB, Davidson MW, Jin Y, Ellisman MH, Tsien RY. A genetically encoded tag for correlated light and electron microscopy of intact cells, tissues, and organisms. PLoS Biol. 2011; 9(4):e1001041.

54. Trost A, Schroedl F, Marschallinger J, Rivera FJ, Bogner B, Runge C, CouillardDespres S, Aigner L, Reitsamer HA. Characterization of dsRed2-positive cells in the doublecortin-dsRed2 transgenic adult rat retina. Histochem Cell Biol. 2014;142(6):601-17.

55. Scalabrino ML, Boye SL, Fransen KM, Noel JM, Dyka FM, Min SH, Ruan Q, de Leeuw CN, Simpson EM, Gregg RG, et al. Intravitreal delivery of a novel AAV vector targets $\mathrm{ON}$ bipolar cells and restores visual function in a mouse model of complete congenital stationary night blindness. Hum Mol Genet. 2015;24(21):6229-39.

56. Ueki Y, Wilken MS, Cox KE, Chipman L, Jorstad N, Sternhagen K, Simic M, Ullom K, Nakafuku M, Reh TA. Transgenic expression of the proneural transcription factor Ascl1 in Muller glia stimulates retinal regeneration in young mice. Proc Natl Acad Sci U S A. 2015;112(44):13717-22.

57. Corso-Díaz X, Simpson EM. Nr2e1 regulates retinal lamination and the development of Muller glia, S-cones, and glycineric amacrine cells during retinogenesis. Mol Brain. 2015;8(37):1-21.

58. Miyawaki T, Uemura A, Dezawa M, Yu RT, Ide C, Nishikawa S, Honda Y, Tanabe $Y$, Tanabe T. TlX, an orphan nuclear receptor, regulates cell numbers and astrocyte development in the developing retina. J Neurosci. 2004; 24(37):8124-34.

59. Rosenbloom KR, Armstrong J, Barber GP, Casper J, Clawson H, Diekhans M, Dreszer TR, Fujita PA, Guruvadoo L, Haeussler M, et al. The UCSC Genome Browser database: 2015 update. Nucleic Acids Res. 2015:43(Database issue): D670-681.

60. ENCODE Project Consortium. An integrated encyclopedia of DNA elements in the human genome. Nature. 2012;489(7414):57-74.

61. ENCODE Project Consortium. The ENCODE (ENCyclopedia Of DNA Elements) Project. Science. 2004;306(5696):636-40.

62. Thomas DJ, Rosenbloom KR, Clawson H, Hinrichs AS, Trumbower H, Raney BJ, Karolchik D, Barber GP, Harte RA, Hillman-Jackson J, et al. The ENCODE Project at UC Santa Cruz. Nucleic Acids Res. 2007;35(Database issue):D663-667.

63. Rosenbloom KR, Dreszer TR, Pheasant M, Barber GP, Meyer LR, Pohl A, Raney BJ, Wang T, Hinrichs AS, Zweig AS, et al. ENCODE whole-genome data in the UCSC Genome Browser. Nucleic Acids Res. 2010;38(Database issue): D620-625.

64. Rosenbloom KR, Sloan CA, Malladi VS, Dreszer TR, Learned K, Kirkup VM, Wong MC, Maddren M, Fang R, Heitner SG, et al. ENCODE data in the UCSC Genome Browser: year 5 update. Nucleic Acids Res. 2013;41(Database issue): D56-63.

65. Rosenbloom KR, Dreszer TR, Long JC, Malladi VS, Sloan CA, Raney BJ, Cline MS, Karolchik D, Barber GP, Clawson H, et al. ENCODE whole-genome data in the UCSC Genome Browser: update 2012. Nucleic Acids Res. 2012; 40(Database issue):D912-917.

66. ENCODE. Project Consortium: A user's guide to the encyclopedia of DNA elements (ENCODE). PLoS Biol. 2011;9(4):e1001046.

67. Griffith OL, Montgomery SB, Bernier B, Chu B, Kasaian K, Aerts S, Mahony S, Sleumer MC, Bilenky M, Haeussler M, et al. ORegAnno: an open-access community-driven resource for regulatory annotation. Nucleic Acids Res. 2008;36(Database issue):D107-113.

68. Montgomery SB, Griffith OL, Sleumer MC, Bergman CM, Bilenky M, Pleasance ED, Prychyna Y, Zhang X, Jones SJ. ORegAnno: an open access database and curation system for literature-derived promoters, transcription factor binding sites and regulatory variation. Bioinformatics. 2006;22(5):637-40.

69. Anderson PJ, Watts HR, Jen S, Gentleman SM, Moncaster JA, Walsh DT, Jen LS. Differential effects of interleukin-1 beta and S100B on amyloid precursor protein in rat retinal neurons. Clin Ophthalmol. 2009;3:235-42. 